\title{
Recovering Exponential Accuracy From Non-Harmonic Fourier Data Through Spectral Reprojection
}

\author{
Anne Gelb*Taylor Hines ${ }^{\dagger}$
}

November 15, 2010

\begin{abstract}
Spectral reprojection techniques make possible the recovery of exponential accuracy from the partial Fourier sum of a piecewise-analytic function, essentially conquering the Gibbs phenomenon for this class of functions. This paper extends this result to non-harmonic partial sums, proving that spectral reprojection can reduce the Gibbs phenomenon in nonharmonic reconstruction as well as remove reconstruction artifacts due to erratic sampling. We are particularly interested in the case where the Fourier samples form a frame. These techniques are motivated by a desire to improve the quality of images reconstructed from non-uniform Fourier data, such as magnetic resonance (MR) images.
\end{abstract}

Keywords: Fourier frames, piecewise-analytic functions; non-harmonic reconstruction; Gibbs phenomenon; Gegenbauer reconstruction.

\section{Introduction}

When attempting to reconstruct a piecewise-analytic function with Fourier data, it is well known that the Gibbs phenomenon leads to

\footnotetext{
*anne.gelb@asu.edu. School of Mathematical and Statistical Sciences, Arizona State University, P.O. Box 871804.

${ }^{\dagger}$ thines@math.purdue.edu. Department of Mathematics, Purdue University, 150 N. University Street, West Lafayette, IN 47907-2067.
} 
spurious oscillations at the jump discontinuities as well as a reduced (to first-order) rate of convergence elsewhere, [13]. Although there have been many efforts to resolve these reconstruction artifacts, of particular interest in this investigation is the spectral reprojection method. Specifically, we will investigate the Gegenbauer reconstruction method.

Since its introduction in 1992, the Gegenbauer reconstruction method has been used to resolve the Gibbs phenomenon for piecewiseanalytic functions within the regions of smoothness, [15, 14]. Suppose we are given a finite number of Fourier coefficients of a piecewiseanalytic function, that is, we are able to project the function onto a finite family of Fourier basis functions. The spectral reprojection method reprojects this corresponding Fourier partial sum approximation within a smooth subinterval onto a more 'suitable' family of functions (in this case, Gegenbauer polynomials). In particular, the high Fourier modes have exponentially small reprojection onto the Gegenbauer polynomials, and hence the high frequency oscillations that would normally appear at the internal boundaries of each smooth subinterval are erased. The reprojection yields exponential accuracy (improved from first order) on a much smaller expansion. This method has been implemented with great success when attempting to improve numerical approximations given only a finite number of Fourier coefficients (see [14] and references therein).

In several applications, however, such as magnetic resonance imaging (MRI), data may be collected as a series of non-uniform Fourier coefficients (see $[2,21,25,26]$ ). Therefore, standard Fourier reconstruction methods cannot be straightforwardly applied. Instead, the reconstruction techniques commonly used tend to be adhoc, and vary between fields. In the particular case of MR imaging, several techniques were designed to achieve a more accurate image reconstruction, (see e.g. [21]), but no systematic study of convergence analysis was performed until [26].

This paper takes a different approach. We expand on an idea introduced in [7], where the authors extend certain "desirable" properties of the standard Fourier basis to families of nonharmonic complex exponentials called Fourier frames. We pause to note that the sampling sequences commonly used in MRI and other applications are often not in fact frames. However, our numerical results strongly 
suggest that the same techniques designed for frames can be utilized under less stringent conditions. For more information on designing MRI sampling trajectories which conform to the conditions of a Fourier frame, see [2] and other papers by these authors.

Unfortunately, finite-dimensional approximations involving Fourier frames share many of the same undesirable properties as standard partial Fourier approximations. Namely, convergence rates are severely reduced for discontinuous (or nonperiodic) functions with spurious oscillations appearing at the points of discontinuity. Figure 4 displays this type of behavior. In addition, frames may sometimes exhibit growth at the interval boundaries leading to a Runge-like phenomenon. In fact, due in part to the great deal of freedom allowed when constructing Fourier frames, establishing convergence results for general frames is impractical. As a result, the numerical effectiveness of different Fourier frames can vary wildly, and the observed reconstruction difficulties are not surprising.

As will be shown in this paper, the spectral reprojection method implemented in the uniform Fourier case can be generalized as one way of overcoming the non-uniform reconstruction difficulties. In particular, we demonstrate that finite-dimensional Fourier frame approximations of a piecewise-analytic function can be reprojected onto Gegenbauer polynomials in order to recover a pointwise exponentially accurate reconstruction everywhere in the domain, thereby generalizing the result in [15] to nonharmonic Fourier approximations.

This paper is organized as follows. In Section 2 we review Fourier reconstruction techniques for piecewise-analytic functions, including standard filtering and the Gegenbauer reconstruction method as it was introduced in [15]. In Sections 3 and 4 we introduce Fourier frames, and give several fundamental results about representation and reconstruction with these families. In Section 5 we demonstrate how spectral reprojection methods can be extended to non-uniform frames of exponentials. Numerical examples are provided in Section 6, and Section 7 contains some concluding remarks and a discussion about future investigations. 


\section{Fourier reconstruction methods for piecewise- analytic functions}

Consider a real-valued function $f \in L^{2}[-1,1]$, and suppose that we are given the first $2 N+1$ Fourier coefficients of $f$, denoted $\hat{f}(-N), \ldots, \hat{f}(N)$, where

$$
\hat{f}(n)=\left\langle f(x), e^{i \pi n x}\right\rangle=\frac{1}{2} \int_{-1}^{1} f(x) e^{-i \pi n x} d x .
$$

It is well known that if $f$ is analytic and periodic, then the partial Fourier sum

$$
\left(S_{N} f\right)(x)=\sum_{n=-N}^{N}\left\langle f(x), e^{i \pi n x}\right\rangle e^{i \pi n x}
$$

rapidly converges (uniformly) to the original function $f$. However, if $f$ has discontinuities or is nonperiodic, this reconstruction is reduced to first-order convergence and at the discontinuities (or endpoints) the reconstruction suffers $\mathcal{O}(1)$ oscillations whose magnitude does not decrease (the Gibbs phenomenon). Figure 1 displays an example of using (2) to approximate the function $f(x)=x$.

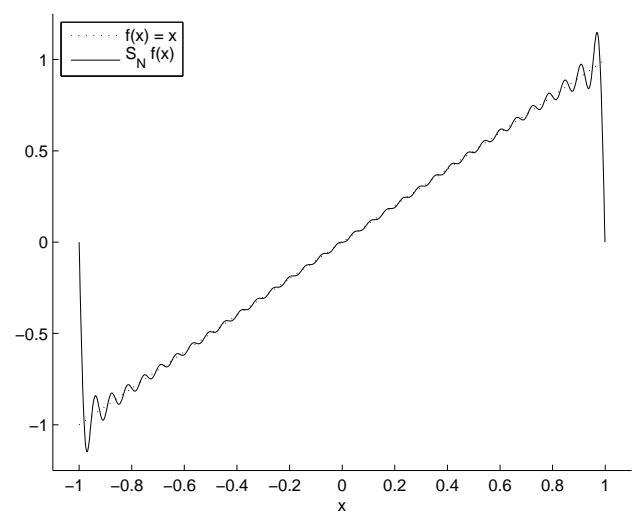

Figure 1: The Fourier partial sum, (2), for $f(x)=x$ with $N=32$. 
It will be useful to write the Fourier partial sum, (2), as $S_{N} f=$ $f * D_{N}$, where $D_{N}(x)$ is the Dirichlet kernel

$$
D_{N}(x)=\sum_{n=-N}^{N} e^{i n x},
$$

the finite Fourier approximation of the delta function.

\section{$2.1 \quad$ Filtering}

The most practical way to mitigate oscillations and improve the order of convergence is to apply a spectral filter. The effect of filtering is to essentially "smooth out" the function reconstruction, which improves accuracy in the analytic regions. Unfortunately, the reduced resolution at high frequencies causes smearing at any points of discontinuity, and therefore any subsequent processing can be severely affected. However, since filtering is such a widely used approach, we include a brief review here as well as a discussion about extending filters to the case of non-uniform Fourier coefficients in Section 5.1.

Definition 1 [19] A filter of order $p$ is an even $C^{p-1}$ function $\sigma$ : $\mathbb{R} \rightarrow \mathbb{R}$ with the property that

i. $\sigma(\xi)=0$ for all $|\xi|>1$

ii. $\sigma(0)=1$ and $\sigma(1)=0$.

iii. $\sigma^{(k)}(0)=\sigma^{(k)}(1)=0$ for all $1 \leq k \leq p-1$.

Two common examples are the second-order Lanczos filter (or sinc filter)

$$
\sigma(\xi)=\frac{\sin \pi \xi}{\pi \xi}
$$

and the exponential filter of order $p$

$$
\sigma(x)= \begin{cases}1 & \text { if }|\xi| \leq \xi_{0} \\ e^{-\gamma \xi^{p}} & \text { if }|\xi|>\xi_{0}\end{cases}
$$

where the parameter $\gamma$ is chosen so that $\sigma(1) \approx 0$. 
In essence, a filter improves the decay rate of expansion coefficients so that the modified expansion

$$
S_{N}^{\sigma} f=\sum_{n=-N}^{N} \sigma\left(\frac{|n|}{N}\right) \hat{f}_{n} e^{i \pi n x}
$$

converges more quickly to the (filtered) original function in regions of smoothness. For a more in-depth discussion of filtering as well as specific results about the convergence rate of filtered Fourier expansions, see [19].

Filtering has the advantage of simple construction and numerical implementation. The FFT can be directly applied to (6). Although filtering reduces the Gibbs phenomenon, it does not eliminate it completely. As demonstrated in Figure 2, some oscillations (or blurring for low order filters) remain at the discontinuities and the convergence rate away from the discontinuities does not improve uniformly. Hence many alternative approaches have been introduced (e.g. $[8,10,16,22,23,24])$ to reduce the effects of the Gibbs phenomenon. Below we discuss the Gegenbauer reconstruction method, [15], as one way of overcoming the Gibbs phenomenon without sacrificing accuracy at the points of discontinuity.

\subsection{Gegenbauer reconstruction}

Unlike the partial Fourier sum, approximations by certain classes of orthogonal polynomials are exponentially accurate for (nonperiodic) analytic functions, [13]. In [15] it was shown that it is possible to obtain a (pointwise) exponentially accurate approximation of an analytic function $f$ by reprojecting the partial Fourier sum of $f$ onto a suitable family of orthogonal polynomials, namely, the Gegenbauer polynomials. For ease of presentation we will assume that $f$ is analytic but not periodic. The spectral reprojection method can also be applied to piecewise-analytic functions, since a linear change of variables makes it possible to consider each region of analyticity separately using the theory established for nonperiodic analytic functions, [14].

The Gegenbauer polynomials form an orthogonal basis in the interval $[-1,1]$ with respect to the weight function $\left(1-x^{2}\right)^{\mu-\frac{1}{2}}$, for 
$\mu \geq 0$. The Gegenbauer polynomial of degree $m$ is denoted $C_{m}^{\mu}(x)$. Given a function $f \in L^{2}[-1,1]$, the $m^{\text {th }}$ Gegenbauer coefficient is given by

$$
\hat{f}^{\mu}(m)=\frac{1}{h_{m}^{\mu}}<f, C_{m}^{\mu}>=\frac{1}{h_{m}^{\mu}} \int_{-1}^{1}\left(1-x^{2}\right)^{\mu-\frac{1}{2}} f(x) C_{m}^{\mu}(x) d x,
$$

where $h_{m}^{\mu}=\int_{-1}^{1}\left(1-x^{2}\right)^{\mu-\frac{1}{2}} C_{m}^{\mu}(x) C_{m}^{\mu}(x) d x=\sqrt{\pi} C_{m}^{\mu}(1) \frac{\Gamma\left(\mu+\frac{1}{2}\right)}{\Gamma(\mu)(m+\mu)}$ and the weight function $\omega_{\mu}(x)=\left(1-x^{2}\right)^{\mu-\frac{1}{2}}$.

The general reprojection process is as follows. Given the first $2 N+1$ Fourier coefficients $\hat{f}(n)$ as defined in (1), of an analytic (but not periodic) function $f$ :

i. Construct the partial Fourier sum, $(2), S_{N} f=\sum_{n=-N}^{N} \hat{f}(n) e^{i n x}$.

ii. Calculate the first $M=\lfloor\beta N\rfloor, \beta<1$ Gegenbauer coefficients, (7), of $S_{N} f,(2)$. Note that step (ii.) can be calculated efficiently using (26), as shown in $[9, \mathrm{p} .213(7)]$. In fact, one advantage of the Gegenbauer reconstruction method is that the closed form in (26) allows for direct use of the FFT, [14].

iii. Reconstruct $f$ as the partial sum

$$
P_{M}^{\mu} S_{N} f=\sum_{m=1}^{M}\left(\widehat{S_{N} f}\right)^{\mu}(m) C_{m}^{\mu}(x) .
$$

A simple application of the triangle inequality shows that the total reconstruction error for a function $f \in L^{2}[-1,1]$ after reprojecting the partial Fourier approximation, (2), onto Gegenbauer polynomials, (8), can be estimated as

$$
\left\|f-P_{M}^{\mu} S_{N} f\right\|_{\infty} \leq\left\|f-P_{M}^{\mu} f\right\|_{\infty}+\left\|P_{M}^{\mu} f-P_{M}^{\mu} S_{N} f\right\|_{\infty} .
$$

The first term on the right-hand side has been called the regularization error, and the second term has been referred to as the truncation error. A theorem in [15, Thm. 5.1, p.91] proves that both are exponentially small when the parameters $\mu$ and $M$ are chosen to be large enough, for example, by setting $\mu=\alpha N$ and $M=\beta N$ for $\alpha$ and $\beta$ with $0<\alpha, \beta<1$. Therefore, a (pointwise) exponentially accurate recovery of $f$ is possible everywhere. Figure 2 demonstrates the Gegenbauer reconstruction method for the function $f(x)=x$. 

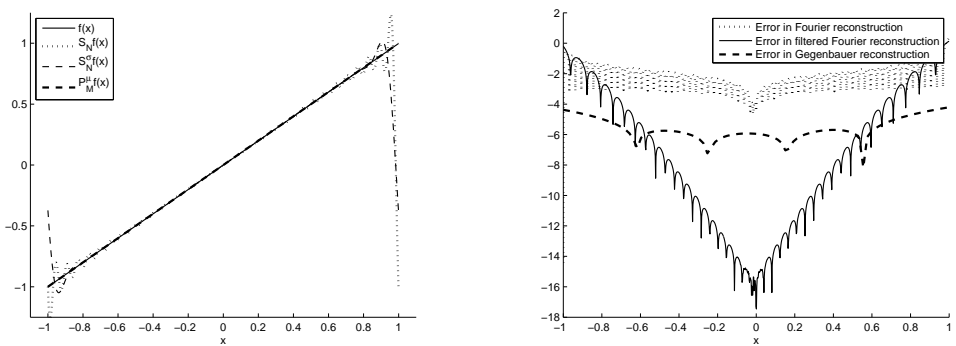

Figure 2: (left) Reconstruction of $f(x)=x$ using fourth-order filtering (5), and the Gegenbauer reconstruction method, (8). (right) The logarithmic error comparison. Here, $N=32, M=4$, and $\mu=4$.

\section{$3 \quad$ Frames of exponentials}

In several physical applications (e.g. magnetic resonance imaging) a function may be sampled at non-uniform Fourier modes. That is, suppose we are given samples of the form

$$
\hat{f}\left(\lambda_{n}\right)=\int_{-1}^{1} f(x) e^{-i \lambda_{n} x} d x
$$

where the family $\left\{\lambda_{n}\right\}_{n \in \mathbb{Z}}$ of real numbers are not necessarily integers. These sampling schemes, (11)-(13), displayed in Figure 3, are motivated by some possible applications. In particular, (11) represents a one-dimensional analogue of the spiral sampling that is sometimes used in MRI [2]. We note that because the high frequencies are sampled so sparsely under this scheme, the corresponding family $\left\{e^{i \lambda_{n} x}\right\}$ is not complete in $L^{2}(I)$ for any interval $I$, and so cannot form a frame. However, this case is still of high interest, since it is the $1 \mathrm{D}$ analogue of a spiral, which is a commonly-studied MRI sampling scheme. Jittered sampling, (12) and (13), reflects the common situation when sensing equipment may err in collecting uniform samples. Figure 3 compares sampling schemes (11) and (13) to the uniform case. 
$\left\{\lambda_{n}\right\}$ spaced logarithmically, centered around 0 .

$\left\{\lambda_{n}\right\}=\left\{n+\nu_{1 / 4}\right\}$, uniform distribution $\nu_{1 / 4}$ of magnitude $\leq 1 / 4$.

$\left\{\lambda_{n}\right\}=\left\{n+\nu_{1 / 2}\right\}$, uniform distribution $\nu_{1 / 2}$ of magnitude $\leq 1 / 2$.

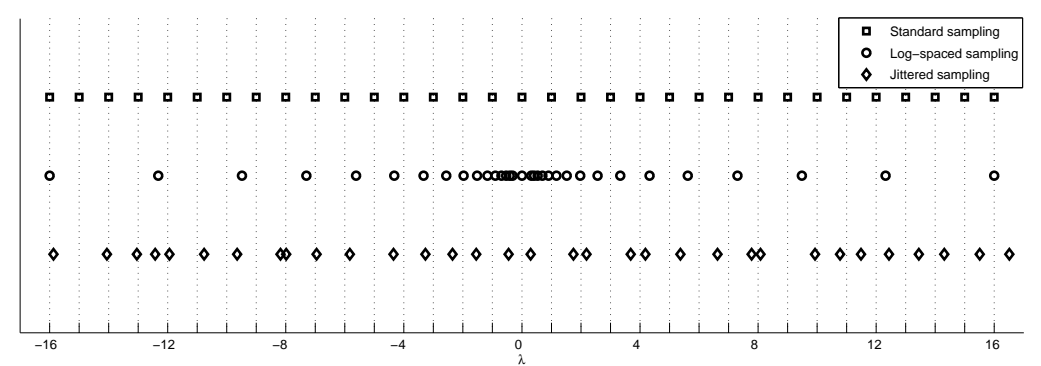

Figure 3: An example of different Fourier sampling sequences $\left\{\lambda_{n}\right\}_{n=-25}^{25}$ as compared to standard Fourier sampling.

For an arbitrary piecewise-analytic function $f$, if we are only given the data $\left\{\hat{f}\left(\lambda_{n}\right)\right\}_{n=-N}^{N}$ for some non-uniform family $\left\{\lambda_{n}\right\}_{n=-N}^{N}$ in $\mathbb{R}$, it is unclear whether the original signal $f$ can be meaningfully reconstructed at all, let alone whether or not any reprojection method will help resolve any existing reconstruction artifacts stemming from jump discontinuities. For this reason, we now turn to the theory of Fourier frames, [7], which were developed in 1952 as a way of generalizing the techniques of classical Fourier analysis. In what follows, we demonstrate that the theory of Fourier frames can be extended to incorporate spectral reprojection methods.

More specifically, let $\left\{\lambda_{n}\right\}_{n \in \mathbb{Z}}$ be a family in $\mathbb{R}$, and denote by $L^{2}(I)$ the Hilbert space. Here we use the standard inner product $\langle f, g\rangle=\int_{I} f(x) \bar{g}(x) d x$ and denote by $\|\cdot\|$ the norm on $L^{2}(I)$ induced by this inner product of square-integrable functions on an interval $I$.

Definition 2 The family $\left\{e^{i \lambda_{n} x}\right\}_{n \in \mathbb{Z}}$ in $L^{2}(I)$ is called a Fourier frame (or simply a frame) if there exist constants $B \geq A>0$ such 
that

$$
A\|f\|^{2} \leq \sum_{n \in \mathbb{Z}}\left|\left\langle f(x), e^{i \lambda_{n} x}\right\rangle\right|^{2} \leq B\|f\|^{2}
$$

for all $f \in L^{2}(I)$. The constants $A$ and $B$ are called the frame bounds, and the frame is called tight if $A=B$.

Note that (14), called the frame condition, is simply a generalization of Parseval's identity for orthonormal bases to an inequality.

Example 1 The standard Fourier basis $\left\{\frac{1}{\sqrt{2 \pi}} e^{i n x}\right\}_{n \in \mathbb{Z}}$ is a frame for $L^{2}(-\pi, \pi)$. It follows then that the set $\left\{e^{i n x}\right\}$ is a tight frame with bounds $A=B=2 \pi$.

It is important to realize that a Fourier frame need not be orthogonal, or even linearly independent:

Example 2 The family with repeated entry $e^{i x}$ :

$$
\left\{\ldots, e^{-2 i x}, e^{-i x}, 1, e^{i x}, e^{i x}, e^{2 i x}, e^{3 i x}, \ldots\right\}
$$

is a frame with bounds $A=2 \pi$ and $B=4 \pi$.

In general, it is often convenient (although not always accurate) to think of a frame as an "overcomplete basis."

As shown in [7], a Fourier frame possesses many of the desirable properties of the Fourier basis. In particular, a function can be represented and reconstructed exactly by its frame samples. First, notice that the lower bound in the frame condition, (14), ensures that the family $\left\{e^{i \lambda_{n} x}\right\}$ is complete in $L^{2}(I)$, since if there existed a nonzero $f \in \operatorname{span}\left\{e^{i \lambda_{n} x}\right\}^{\perp}$, then we would have $0<A\|f\|^{2} \leq 0$, a contradiction. To see that representation and reconstruction are possible with a frame, consider the "naive" reconstruction operator $S: L^{2}(I) \rightarrow L^{2}(I)$ defined by $S f=\sum_{n \in \mathbb{Z}}\left\langle f(x), e^{i \lambda_{n} x}\right\rangle e^{i \lambda_{n} x}$. The upper bound in the frame condition ensures that the sampling sequence $\left\{\left\langle f(x), e^{i \lambda_{n} x}\right\rangle\right\}_{n \in \mathbb{Z}} \in \ell^{2}(\mathbb{Z})$ and hence this operator is well-defined. In general, this will not give a good reconstruction, since we do not expect this operator to give an orthonormal basis expansion. However, the following fundamental result demonstrates how this operator can be used in a meaningful way. 
Theorem 1 (The frame operator) [7] If $\left\{e^{i \lambda_{n} x}\right\}_{n \in \mathbb{Z}}$ is a frame for $L^{2}(I)$, then the operator $S: L^{2}(I) \rightarrow L^{2}(I)$ defined by $S f=$ $\sum_{n \in \mathbb{Z}}\left\langle f(x), e^{i \lambda_{n} x}\right\rangle e^{i \lambda_{n} x}$ is bounded, invertible, positive, and self-adjoint.

Clearly $S$ is bounded and positive by the frame condition, (14). Below we demonstrate that $S$ is a bijection. It is then straightforward to show that $S$ is invertible and self-adjoint, and we refer readers to [5] for a detailed proof.

To show $S$ is injective, first note that for all $f \in L^{2}(I)$, we have $\langle S f, f\rangle=\left\langle\sum_{n}\left\langle f, e^{i \lambda_{n} x}\right\rangle e^{i \lambda_{n} x}, f\right\rangle=\sum_{n}\left\langle f, e^{i \lambda_{n} x}\right\rangle \overline{\left\langle f, e^{i \lambda_{n} x}\right\rangle}=\sum_{n}\left|\left\langle f, e^{i \lambda_{n} x}\right\rangle\right|^{2}$.

Therefore, if $S f=0$, then $\sum_{n}\left|\left\langle f, e^{i \lambda_{n} x}\right\rangle\right|^{2}=0$, and hence $\|f\|=0$ by the frame condition.

To show $S$ is surjective, assume that there is some $g \in \operatorname{ran} S^{\perp}$. But then $\langle S f, g\rangle=0$ for all $f \in L^{2}(I)$, particularly, $\langle S g, g\rangle=0$. Therefore, $g=0$ by the frame condition, and so $\operatorname{ran} S=L^{2}(I)$.

Due to its importance, we call this operator the (Fourier) frame operator associated with $\left\{e^{i \lambda_{n} x}\right\}$. When the particular frame is clear from context, we simply call $S$ the frame operator.

As an immediate result of Theorem 1, we obtain (by self-adjointness) the identity

$$
f=S S^{-1} f=\sum_{n \in \mathbb{Z}}\left\langle f(x), S^{-1} e^{i \lambda_{n} x}\right\rangle e^{i \lambda_{n} x}
$$

which shows that $f$ can be represented (or analyzed) by the sequence $\left\{\left\langle f(x), S^{-1} e^{i \lambda_{n} x}\right\rangle\right\}_{n \in \mathbb{Z}}$ in $\ell^{2}(\mathbb{Z})$. Similarly, we obtain

$$
f=S^{-1} S f=\sum_{n \in \mathbb{Z}}\left\langle f(x), e^{i \lambda_{n} x}\right\rangle S^{-1} e^{i \lambda_{n} x}
$$

which demonstrates how $f$ can be reconstructed (or synthesized) exactly from its frame coefficients.

A fundamental result about frames is that if $\left\{e^{i \lambda_{n} x}\right\}$ is a frame for $L^{2}(I)$ with bounds $A \leq B$, then the functions $\left\{S^{-1} e^{i \lambda_{n} x}\right\}$ form a frame for $L^{2}(I)$ with bounds $1 / B, 1 / A$, called the (canonical) dual frame, [5]. Furthermore, it follows that $S^{-1}$ is the frame operator associated with the dual frame and

$$
1 / B \leq\left\|S^{-1}\right\| \leq 1 / A .
$$


As shown in (16), every function can be expanded as a sum of dual frame functions if the frame coefficients are known. However, this expansion will not be unique in general. In fact, there are usually many different ways of writing out the frame expansion of a function since the frame functions (as well as the dual frame functions) are not linearly independent.

In practice, we only deal with a finite number of frame functions, and hence we must be satisfied with a finite-dimensional approximation. Furthermore, there is no closed form equation for constructing the inverse frame operator, which makes this theoretical result computationally infeasible. For this reason, several methods have been developed to numerically construct a finite-dimensional frame approximation.

\section{Reconstruction with frames of exponentials}

Because finite frame expansions are the only practical application of this theory, it is important to study the properties of finitedimensional frame approximations. Consider the operator

$$
S_{N} f=\sum_{n=-N}^{N}\left\langle f(x), e^{i \lambda_{n} x}\right\rangle e^{i \lambda_{n} x}
$$

which, if this frame is the standard Fourier basis, is a direct generalization of a partial Fourier sum, (2). First, notice that if $\left\{e^{i \lambda_{n} x}\right\}_{n \in \mathbb{Z}}$ is a frame for $L^{2}(I)$ then $\left\{e^{i \lambda_{n} x}\right\}_{n=-N}^{N}$ is a frame for its (linear) span. It is not hard to see that the frame operator associated with this finite subfamily is exactly (18). Therefore it follows that $S_{N}$ is invertible (on $\operatorname{span}\left\{e^{i \lambda_{n} x}\right\}_{n=-N}^{N}$ ) and that $S_{N} \rightarrow S$ uniformly as $N \rightarrow \infty$ (see [6], for example). We call the operator $S_{N}$ the finite frame operator associated with the frame $\left\{e^{i \lambda_{n} x}\right\}_{n \in \mathbb{Z}}$. A theorem in [4, Thm. 3, p.83 ] proves that $S_{N}^{-1} \rightarrow S^{-1}$ strongly as $N \rightarrow \infty$, and hence finite-dimensional approximations of the frame operator are possible.

In particular, we can see from (18) that the operator

$$
T_{N} f=\sum_{n=-N}^{N}\left\langle f(x), e^{i \lambda_{n} x}\right\rangle S_{N}{ }^{-1} e^{i \lambda_{n} x},
$$


called the finite frame approximation, is exactly the projection of $f$ onto $\operatorname{span}\left\{e^{i \lambda_{n} x}\right\}_{n=-N}^{N}$. However, we are faced here with the identical problem as before, namely, that the inverse (finite) frame operator is computationally infeasible to construct. Fortunately, there was given in [7] an iterative algorithm for calculating these expansions provided only with the frame coefficients.

Theorem 2 (The Frame Algorithm) [7] Let $\left\{e^{i \lambda_{n} x}\right\}_{n \in \mathbb{Z}}$ be a (Fourier) frame for $L^{2}(I)$ with bounds $A, B>0$, and let $f \in L^{2}(I)$. Iteratively define the functions $f_{k}, k \in \mathbb{N}$ by

$$
\begin{aligned}
f_{0} & =0 \\
f_{k} & =f_{k-1}+\frac{2}{A+B} S\left(f-f_{k-1}\right)
\end{aligned}
$$

for $k \geq 1$. Then $f_{k} \rightarrow f$ with error bounds

$$
\left\|f_{k}-f\right\| \leq\left(\frac{B-A}{B+A}\right)^{k}\|f\|
$$

The proof of this theorem essentially relies on the fact that $S$ is positive and that $\|I-S\| \leq \frac{B-A}{B+A} \leq 1$, which follows from the frame condition, (14), and Theorem 1. It is an immediate corollary that the frame algorithm can be applied in the finite-dimensional case as well, by simply replacing $S$ with $S_{N}$. In this case, we have $f_{k} \rightarrow$ $T_{N} f$ as $\left\|f_{k}-T_{N} f\right\| \leq\left(\frac{B_{N}-A_{N}}{B_{N}+A_{N}}\right)^{k}\left\|T_{N} f\right\|$, where $A_{N}$ and $B_{N}$ are the respective lower and upper bounds for the frame $\left\{e^{i \lambda_{n} x}\right\}_{n=-N}^{N}$. Hence the frame algorithm recovers the approximation (19). Figure 4 illustrates the use of the frame algorithm presented in Theorem 2.

Notice that the frame bounds provide useful information, since the convergence of the algorithm is sensitive to the ratio $A / B$, with $A / B \approx 0$ resulting in very slow convergence and $A / B \approx 1$ resulting in fast convergence (see Examples 1 and 2). We also note that the frame algorithm can be generalized somewhat to account for inexact estimates of the frame bounds by replacing the fraction $\frac{2}{A+B}$ in the algorithm by a parameter $\gamma$. However, crude estimates of $\gamma$ (i.e. underestimates and overestimates of $A$ and $B$, respectively) will further decrease the performance of the algorithm, [17]. Several accelerated frame algorithms were developed in [17], which can vastly improve 


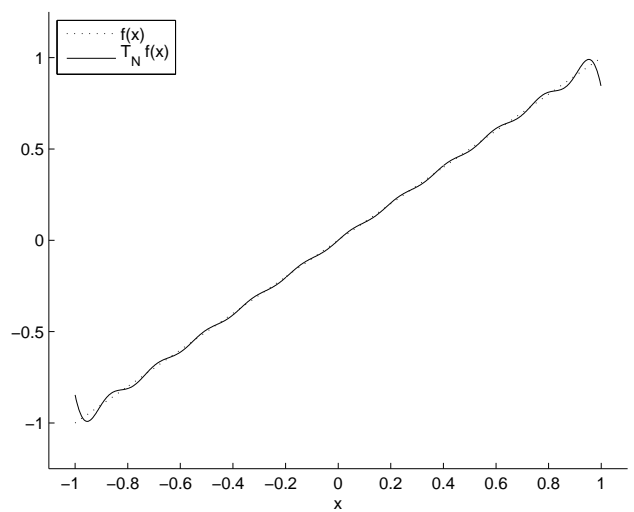

Figure 4: A reconstruction of the function $f(x)=x$ from jittered Fourier frame samples (12) using the frame algorithm (Theorem 2) with $N=32$, to recover $T_{N} f$ in (19).

the convergence of the frame algorithm when the frame bounds are poor. We adopt the (Chebyshev) accelerated frame algorithm [17, Thm. 1, p. 3333] for all of the numerical results and corresponding figures that follow. We direct the reader to [17] for an in-depth analysis, and here only mention that the convergence rate of the accelerated algorithm improves to a relative error of $\left(\frac{\sqrt{B}-\sqrt{A}}{\sqrt{B}+\sqrt{A}}\right)^{k}$, which can decrease the necessary number of iterations by more than an order of magnitude. However, the convergence rate of the frame algorithm still highly depends on the frame bounds, and any underestimates or overestimates of these bounds will severely reduce the rate of convergence. Fortunately, we see that in (19), the only time the inverse frame operator is used is when constructing the dual frame functions. Therefore, if the sampling sequence is known ahead of time, the dual frame can be computed (using the frame algorithm) off-line, reducing the reconstruction time to $\mathcal{O}\left(N^{2}\right)$. Therefore, although the frame algorithm converges slowly for frames with "poor" frame bounds, this slow convergence rate does not necessarily hamper the cost efficiency of he on-line construction.

Finally, while the frame algorithm guarantees convergence in the $L^{2}$ sense, it says nothing about pointwise convergence. This is ev- 
ident in Figure 4 where Theorem 2 is used approximate $f(x)=x$ from the Fourier frame samples in (11). In addition, due to the large amount of freedom that is possible when choosing a frame, analyzing the convergence properties of finite frame approximations is possible for only very special cases (e.g. such as those related to Examples 1 and 2 ). In fact, since a frame can contain arbitrarily many repetitions of the same element, it may yield poor convergence properties. We illustrate some of these issues with the following example:

\section{Example 3}

$$
f(x)= \begin{cases}x+1 & \text { if } x<0 \\ x-1 & \text { if } x \geq 0\end{cases}
$$

Suppose that we are given the Fourier coefficients at jittered points defined by (12). Figure 5 demonstrates that there is a Gibbs phenomenon type of behavior present in non-uniform (frame) reconstructions for piecewise-analytic functions.

In part, the presence of the Gibbs phenomenon in non-uniform reconstructions by frames of exponentials is due to the fact that the sum of the dual frame functions,

$$
K_{N}(x)=\sum_{n=-N}^{N} S^{-1} e^{i \lambda_{n} x}
$$

somewhat resembles the Dirichlet kernel, (3), (see Figures 6 and 7). That is, the projection of the delta function onto the space spanned by a finite number of frame functions is related to the projection onto the space spanned by the same number of Fourier basis functions. This is particularly evident in the jittered sampling case, (12), seen in Figure 6. Because it is this projection that indicates how finite-dimensional Fourier approximations respond to jump discontinuities, we expect the behavior of non-uniform approximations near jump discontinuities to behave similarly to that of the uniform case. Therefore, it is natural to question whether the "nonharmonic Gibbs" phenomenon can be reduced by the same methods that are applied to the uniform case as described in Section 2. In the logsampling case, (11), the majority of the sampling points are clustered at the low frequencies. Hence, we see less of a resemblance of the 

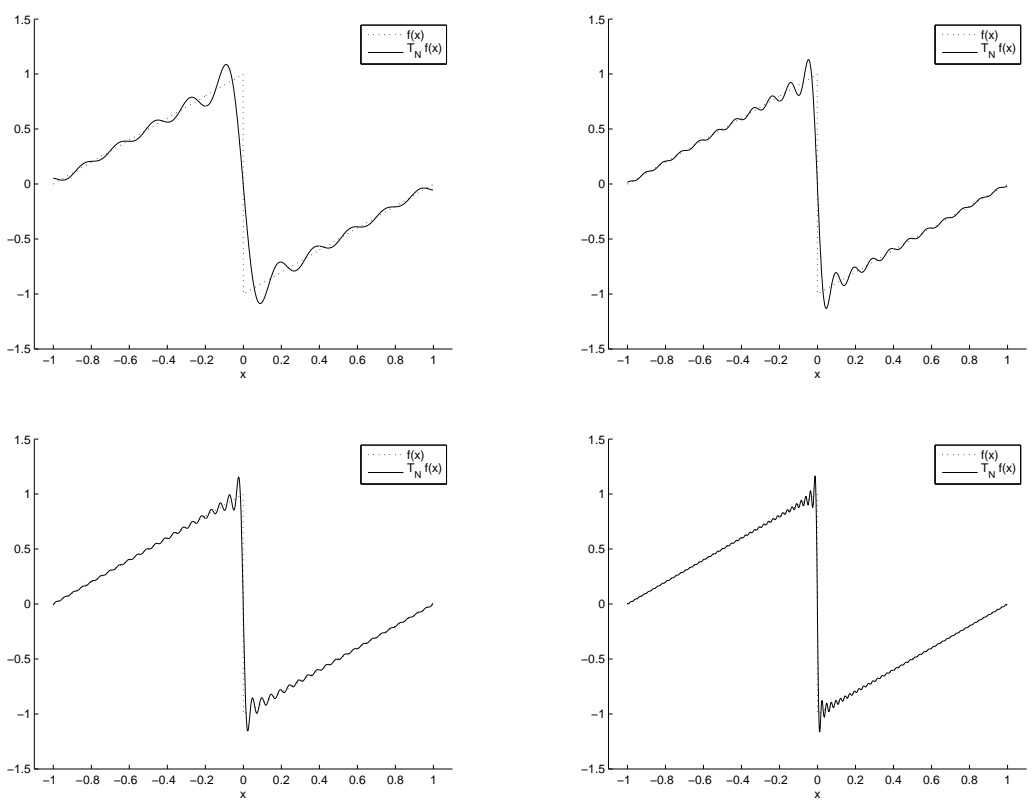

Figure 5: The frame reconstruction, (19), of Example 3 using the jittered Fourier samples, (12). Here we use $N=32,64,128$, and 256.

non-uniform kernel to the Dirichlet kernel (Figure 7) as the frame sampling sequence becomes further removed from the uniform case. Because the high frequencies are sparsely sampled, the log-sampling case performs particularly poorly in response to functions that contain high frequencies (such as functions with jump discontinuities). We also note that in addition to the Gibbs phenomenon-like behavior, we also see a sampling error, which is independent of the analyticity of the function. This error appears to manifest itself more at the boundaries. See [26] for some error analysis due to sampling.

As is clear in Figures 6 and 7, although $K_{N}(x)$ resembles $D_{N}(x)$, convergence $K_{N} \rightarrow D_{N}$ is slow (sub-log if it converges at all), in particular at the boundaries, which is exacerbated by erratic sampling sequenced such as the log-spaced scheme. Because sampling schemes like this are common in some applications, in Section 5 we investigate how the spectral reprojection method might repair re- 

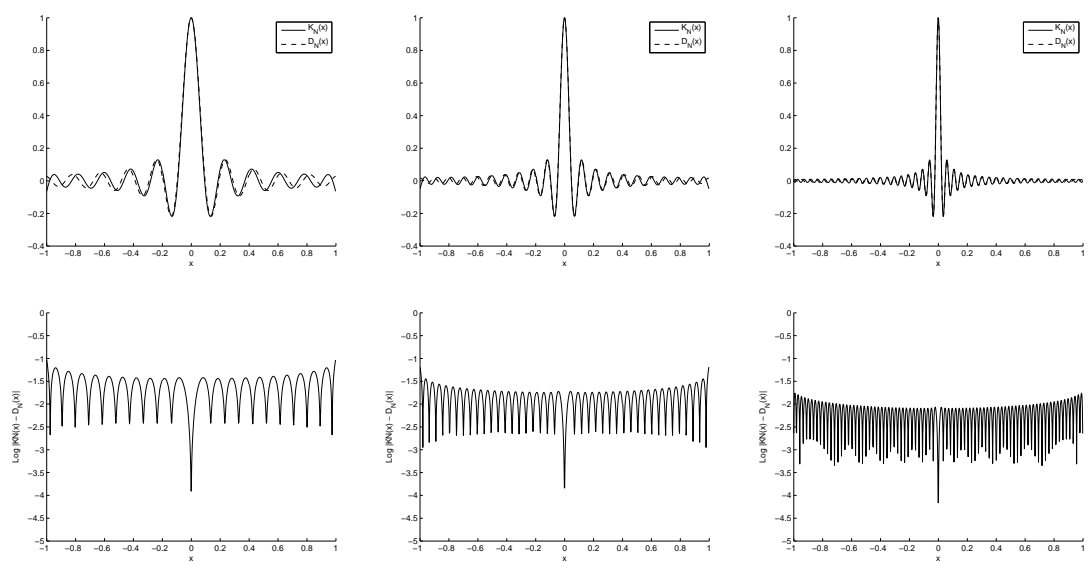

Figure 6: (top) The non-uniform kernel, $K_{N}(x)$, given by (20), as compared to the Dirichlet kernel, $D_{N}(x)$, (3), with $N=32,64$, and 128 , respectively. Here, the sampling sequence is jittered from the uniform case, (12). (bottom) The log error, $\log _{10}\left|K_{N}-D_{N}\right|$, for each $N$.

construction artifacts that are due to sampling error as well as the reconstruction artifacts that similarly exist in the uniform case (such as Gibbs).

\section{$5 \quad$ Reducing reconstruction artifacts for frames of exponentials}

Let $\left\{e^{i \lambda_{n} x}\right\}_{n \in \mathbb{Z}}$ be a frame for $L^{2}[-1,1]$. Suppose $f \in L^{2}[-1,1]$ is analytic (though not necessarily periodic), and that we are given the first $2 N+1$ frame coefficients of $f$. As in (19), denote by $T_{N} f$ the projection of $f$ onto $\operatorname{span}\left\{e^{i \lambda_{n} x}\right\}_{n=-N}^{N}$, which can be calculated iteratively using the frame algorithm introduced in Theorem 2 (or the accelerated version in $[17])$.

\subsection{Filtered frame approximations}

Spectral filtering is a common and straightforward method of reducing the Gibbs phenomenon for partial Fourier expansions of nonperi- 

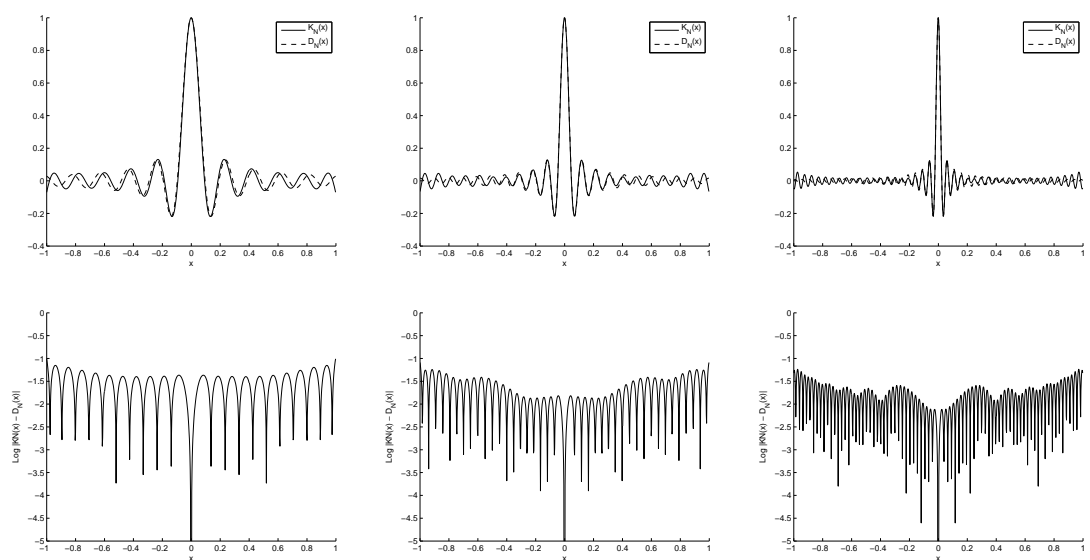

Figure 7: (top) The non-uniform kernel, (20), as compared to the Dirichlet kernel, $D_{N}(x)$, (3), with $N=32,64$, and 128, respectively. Here, the sampling sequence is log-spaced, (11). (bottom) The log error, $\log _{10}\left|K_{N}-D_{N}\right|$, for each $N$.

odic (or piecewise-analytic) functions by improving the convergence rate in the interior of the domain. Hence we modify (19) to construct the filtered frame approximation

$$
T_{N}^{\sigma} f(x)=\sum_{n=-N}^{N}\left\langle f, e^{i \lambda_{n} x}\right\rangle \sigma\left(\lambda_{n}\right) S^{-1} e^{i \lambda_{n} x} .
$$

Unfortunately, spectral filtering methods do not work well for Fourier frame expansions. As shown in Figure 8, the dual frame functions (especially those corresponding to low-frequency frame functions) grow quickly at the boundaries. In fact, this growth may contribute to the poor convergence properties observed in Section 4 (see discussion following Figure 4), in particular yielding similar effects as the Runge phenomenon. The filtered frame expansion is overwhelmed by this boundary growth and the approximation, (21), may in fact be worse. Furthermore, as frame sampling sequences diverge from the uniform case, the dual frame functions look less like standard Fourier basis functions, and hence spectral filtering does not always perform as expected, even in the interior. 


\section{Example 4}

$$
f(x)=\frac{1}{3} x^{4}+x^{3}-2 x^{2}+1 .
$$

Figures 9, 10, and 11 demonstrate the effects of spectral filtering on the frame approximation of $f(x)=x$ and Example 4 respectively. As is evident in these figures, the filtered frame approximation actually performs more poorly than the standard frame approximation, (19), especially at the boundaries.
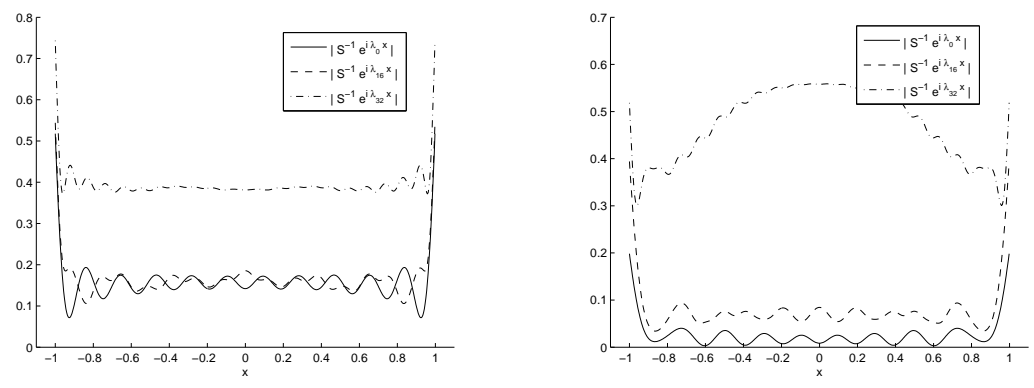

Figure 8: A plot of the modulus of several dual frame functions using a jittered, (13), (left) and log-spaced, (11), (right) sampling sequence with $N=32$.
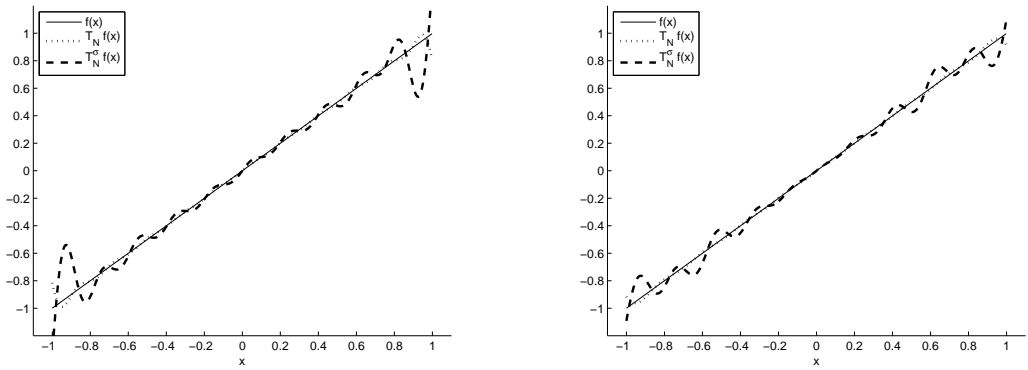

Figure 9: The filtered frame approximation, $(21)$, to $f(x)=x$ using a fourth order filter. (left) Jittered sampling, (13). (right) Log-spaced sampling, (11). Here $N=32$. 

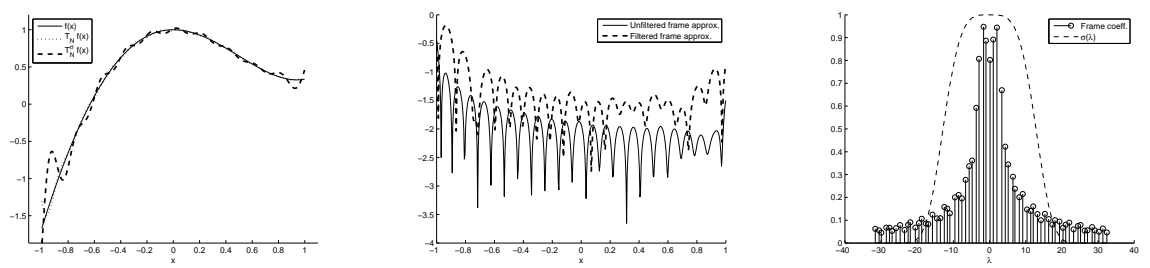

Figure 10: (left) The filtered frame approximation, (21), to Example 4 using jittered sampling, (13), $N=32$. (middle) Comparison of $\log _{10}\left|f-T_{N} f\right|$ and $\log _{10}\left|f-T_{N}^{\sigma} f\right|$. (right) The frame coefficients and the fourth-order filter $\sigma(\lambda),(5)$, are shown to give an idea of the associated decay rate.
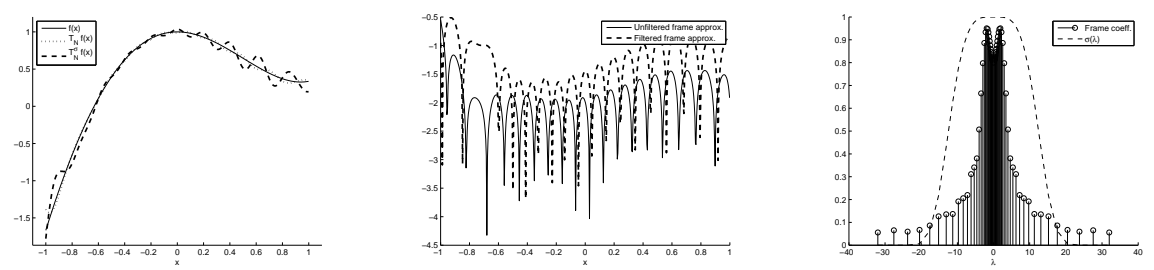

Figure 11: (left) The filtered frame approximation, (21), to Example 4 using log sampling, (11), and $N=32$. (middle) Comparison of $\log _{10}\left|f-T_{N} f\right|$ and $\log _{10}\left|f-T_{N}^{\sigma} f\right|$. (right) The frame coefficients and the fourth-order filter $\sigma(\lambda),(5)$, are shown to give an idea of the associated decay rate.

Straightforward spectral filtering is therefore not the ideal method of reducing reconstruction artifacts for nonharmonic Fourier frame expansions. We now investigate the spectral reprojection method, which may allow us to recover the original function with exponential accuracy everywhere in the domain, including the boundaries.

\subsection{Gegenbauer reprojection with frames}

Our goal is to reproject $T_{N} f$ in (19) onto a finite family of Gegenbauer polynomials, $\left\{C_{m}^{\mu}(x)\right\}_{m=1}^{M}$, in order to recover $f$ pointwise with exponential accuracy. This process is much the same as described in Section 2.2: 
i. Construct the partial frame sum $T_{N} f$, (19), using the iterative algorithm described in Theorem 2 .

ii. Calculate the first $M=\lfloor\beta N\rfloor, \beta<1$ Gegenbauer coefficients, (7), of $T_{N} f,(19)$ :

$$
\widehat{\left(T_{N} f\right)^{\mu}}(m)=\frac{1}{h_{m}^{\mu}} \int_{-1}^{1}\left(1-x^{2}\right)^{\mu-\frac{1}{2}} T_{N} f(x) C_{m}^{\mu}(x) d x .^{1}
$$

iii. Reconstruct $f$ as the partial sum

$$
P_{M}^{\mu} T_{N} f=\sum_{m=1}^{M} \widehat{\left(T_{N} f\right)^{\mu}}(m) C_{m}^{\mu}(x) .
$$

Before proving that exponential accuracy can indeed be recovered by this method, we first review the regularity conditions that can (realistically) be imposed on the sampling sequence $\left\{\lambda_{n}\right\}_{n \in \mathbb{Z}}$.

Definition 3 A sequence $\left\{\lambda_{n}\right\}_{n \in \mathbb{Z}}$ of real numbers is said to have uniform density $d$ if there exists a constant $L \geq 0$ such that $\left|\lambda_{n}-\frac{n}{d}\right| \leq$ $L$ for all $n \in \mathbb{Z}$.

Definition 4 A sequence $\left\{\lambda_{n}\right\}_{n \in \mathbb{Z}}$ of real numbers is called separated (or $\delta$-separated) if there is a constant $\delta>0$ such that $\mid \lambda_{n}-$ $\lambda_{m} \mid>\delta$ for all $m, n \in \mathbb{Z}$. We say that $\left\{\lambda_{n}\right\}$ is relatively separated if it is the union of a finite number of separated sequences.

The following result proves that any sampling sequence which forms a frame must possess some of this structure.

\footnotetext{
${ }^{1}$ The natural quadrature points for computing (23) are the Gegenbauer GaussLobotto points. When reconstructing a piecewise-analytic function using spectral reprojection (see Section 5.3), correct placement of Gauss-Lobotto nodes requires a priori knowledge of the discontinuity locations. Fortunately, however, the weight function $\left(1-x^{2}\right)^{\mu-1 / 2}$ associated with the Gegenbauer polynomials makes the more cost-efficient trapezoidal rule with uniform points a reasonable quadrature choice, [3]. Specifically, the dual frame operator used in (23) can still be computed off-line as before. Our numerical investigations suggest that the difference between the quadrature schemes is negligible.
} 
Theorem 3 (Density criterion for frames) [20, Lem. 2, p.341] If $\left\{e^{i \lambda_{n} x}\right\}_{n \in \mathbb{Z}}$ is a frame for $L^{2}[-1,1]$, then $\left\{\lambda_{n}\right\}_{n \in \mathbb{Z}}$ is the disjoint union of a sequence with uniform density $d \leq \frac{1}{\pi}$ and a relatively separated sequence.

In our main result, Theorem 4, we will show that exponentially accurate recovery through Gegenbauer reprojection is possible for frames whose sampling sequence satisfies some mild regularity conditions. Due to the great deal of freedom allowed by relaxing the orthonormality conditions of the standard Fourier basis, there are some pathological sampling sequences that we wish to avoid. Therefore, we restrict our attention to the following case.

Definition 5 The family $\left\{\lambda_{n}\right\}_{n \in \mathbb{Z}}$ is called a balanced sampling sequence if the following conditions hold:

i. $\left\{e^{i \lambda_{n} x}\right\}$ is a frame for $L^{2}[-1,1]$.

ii. $\left\{\lambda_{n}\right\}$ is $\delta$-separated.

iii. $\left\{\lambda_{n}\right\}$ is increasing, that is, $\lambda_{n} \leq \lambda_{n+1}$ for all $n \in \mathbb{Z}$.

iv. $\left\{\lambda_{n}\right\}$ is balanced, that is, $\lambda_{n} \geq 0$ if $n \geq 0$ and $\lambda_{n}<0$ if $n<0$.

The restriction to frames for $L^{2}[-1,1]$ is just for convenience of presentation, and the results presented here can easily be generalized to any bounded interval. As shown in Theorem 3, there is some regularity imposed on general frames, so Definition 5 applies to a wide variety of Fourier frames. By [5, Thm. 5.1.6, p.91], if $\left\{e^{i \lambda_{n} x}\right\}_{n \in \mathbb{Z}}$ is a frame, then the reconstruction series (16) converges unconditionally. Therefore, we can without loss of generality reorder $\left\{e^{i \lambda_{n} x}\right\}$ so that the sequence $\left\{\lambda_{n}\right\}$ is increasing and balanced. In most practical applications, frequency samples (the frame coefficients) are collected with a high degree of symmetry, and hence it is often justified to assume that the frame coefficients $\hat{f}\left(\lambda_{-N}\right), \ldots, \hat{f}\left(\lambda_{N}\right)$ are "centered" at the low frequencies. We conjecture that our main result, Theorem 4, can be extended to general Fourier frames, although such an extension would provide little additional advantage in most practical applications. 
Because it is of particular interest, we present several results in Section 6 of cases where the sampling sequence does not satisfy Definition 5. Specifically, when data is collected using log-spaced samples, (11), the corresponding family of exponentials is not complete in $L^{2}[-1,1]$ and hence does not form a frame. Therefore, Theorem 4 does not guarantee that Gegenbauer reprojection will deliver exponential convergence. However, we still see improvement in the final reconstruction, which suggests that the Gegenbauer reconstruction method may still be applicable for more general sampling schemes than described in Definition 5.

The following lemma is needed for our main result.

Lemma 1 If $\left\{\lambda_{n}\right\}_{n \in \mathbb{Z}}$ is a balanced sampling sequence, then there is a constant $c>0$ such that

$$
\sum_{|n|>N}\left(\frac{1}{\left|\lambda_{n}\right|}\right)^{\mu} \leq c\left(\frac{1}{\lambda_{ \pm N}}\right)^{\mu-1}
$$

for any positive integer $N$ and any $\mu>2$, where $\lambda_{ \pm N}=\min \left\{\left|\lambda_{N}\right|,\left|\lambda_{-N}\right|\right\}$.

Furthermore, if $\left\{\lambda_{n}\right\}_{n \in \mathbb{Z}}$ has uniform density, then there is a $t>0$ (independent of $N$ or $\mu$ ) such that

$$
\sum_{|n|>N}\left(\frac{1}{\left|\lambda_{n}\right|}\right)^{\mu} \leq c\left(\frac{1}{\pi N-t}\right)^{\mu-1}
$$

for large enough $N$.

Proof First assume that $\left\{\lambda_{n}\right\}_{n \in \mathbb{Z}}$ is a balanced sampling sequence. Note that it suffices to estimate only the right-hand tail of this sum, showing that $\sum_{n>N}\left(\frac{1}{\lambda_{n}}\right)^{\mu} \leq c\left(\frac{1}{\lambda_{N}}\right)^{\mu}$. Since $\delta:=\inf _{n, m}\left\{\left|\lambda_{n}-\lambda_{m}\right|\right\}$, it follows that $\delta \leq \inf _{n}\left\{\left|\lambda_{n-1}-\lambda_{n}\right|\right\}$. Therefore

$$
\sum_{n>N}\left(\frac{1}{\lambda_{n}}\right)^{\mu} \leq \frac{1}{\delta} \sum_{n>N}\left(\frac{1}{\lambda_{n}}\right)^{\mu}\left(\lambda_{n-1}-\lambda_{n}\right) .
$$

By the balanced property of $\left\{\lambda_{n}\right\}$, we know that the function $f(x)=$ $x^{-\mu}$ restricted to the set $\left\{\lambda_{n}\right\}_{n>N}$ is monotonically decreasing. Hence we can estimate the series by the integral $\int_{\lambda_{N}}^{\infty} x^{-\mu} d x=(\mu-1)^{-1} \lambda_{N}^{-(\mu-1)}$. Since $\mu>2$, the result follows by setting $c=1 / \delta$. 
Next, suppose that $\left\{\lambda_{n}\right\}_{n \in \mathbb{Z}}$ has uniform density. Hence there exist constants $d, L>0$ such that $\left|\lambda_{n}-\frac{n}{d}\right| \leq L$ for all $n$. Recall also that Theorem 3 gives $d \leq \frac{1}{\pi}$. Upon taking $N>d L$, we estimate $\sum_{|n|>N}\left(\frac{1}{\lambda_{n}}\right)^{\mu}$. Without loss of generality, it suffices to evaluate this sum over only the positive integers. In this case, we have

$$
\begin{aligned}
\sum_{n>N}\left(\frac{1}{\lambda_{n}}\right)^{\mu} & \leq \sum_{n>N}\left(\frac{1}{\frac{n}{d}-L}\right)^{\mu} \\
& =\sum_{n>N}\left(\frac{d}{n-d L}\right)^{\mu} \\
& \leq c\left(\frac{1}{\pi(N-d L)}\right)^{\mu-1} \\
& =c\left(\frac{1}{\pi N-t}\right)^{\mu-1} .
\end{aligned}
$$

We can now state our main result:

Theorem 4 Let $\left\{e^{i \lambda_{n} x}\right\}_{n \in \mathbb{Z}}$ be frame for $L^{2}[-1,1]$ generated by a balanced sampling sequence, and let $f \in L^{2}[-1,1]$. Then there exists a constant $c>0$ such that the truncation error in (9) satisfies the estimate

$$
\left\|P_{M}^{\mu}\left(f-T_{N} f\right)\right\|_{\infty} \leq c \frac{\Gamma(\mu)(M+\mu) \Gamma(M+2 \mu)}{(M-1) ! \Gamma(2 \mu)}\left(\frac{2}{\lambda_{ \pm N}}\right)^{\mu-1}
$$

for any positive integers $N, M$ and any $\mu>2$.

Proof First, consider the reprojection

$$
P_{M}^{\mu}\left(f-T_{N} f\right)=\sum_{|n|>N}\left\langle f, S^{-1} e^{i \lambda_{n} x}\right\rangle P_{M}^{\mu} e^{i \lambda_{n} x} .
$$

From $[9$, p.213(7)], we have

$$
\frac{1}{h_{m}^{\mu}} \int_{-1}^{1}\left(1-x^{2}\right)^{\mu-\frac{1}{2}} e^{i \lambda_{n} x} C_{m}^{\mu}(x) d x=\Gamma(\mu)\left(\frac{2}{\lambda_{n}}\right)^{\mu} i^{m}(m+\mu) J_{m+\mu}\left(\lambda_{n}\right)
$$


where $J_{\nu}(x)$ is a Bessel function of the first kind. Since $\left\|S^{-1}\right\| \leq 1 / A$, (17), the Cauchy-Schwarz inequality implies that $\left|\left\langle f, S^{-1} e^{i \lambda_{n} x}\right\rangle\right| \leq$ $\|f\| / A$. Therefore,

$$
\begin{aligned}
\left|\left\langle\sum_{|n|<N}\left\langle f, S^{-1} e^{i \lambda_{n} x}\right\rangle e^{i \lambda_{n} x}, C_{m}^{\mu}(x)\right\rangle\right| & \leq \frac{\|f\|}{A} \sum_{|n|<N}\left|\left\langle e^{i \lambda_{n} x}, C_{m}^{\mu}(x)\right\rangle\right| \\
& \leq \frac{\|f\|}{A} \Gamma(\mu)(m+\mu) J_{m+\mu}\left(\lambda_{n}\right)\left|\sum_{|n|<N}\left(\frac{2}{\lambda_{n}}\right)^{\mu}\right| \\
& \leq c \frac{\|f\|}{A} \Gamma(\mu)(m+\mu) J_{m+\mu}\left(\lambda_{n}\right)\left(\frac{2}{\lambda_{ \pm N}}\right)^{\mu-1} \\
& \leq c \frac{\|f\|}{A} \Gamma(\mu)(m+\mu)\left(\frac{2}{\lambda_{ \pm N}}\right)^{\mu-1},
\end{aligned}
$$

where the second line uses (26), the third line applies Lemma 1, and the final line holds since $\left|J_{\nu}(x)\right| \leq 1$ for all $x$. Thus

$$
\begin{aligned}
\left\|P_{M}^{\mu}\left(f-T_{N} f\right)\right\|_{\infty} & \leq M \max _{1 \leq m \leq M}\left\|\left\langle\sum_{|n|<N}\left\langle f, S^{-1} e^{i \lambda_{n} x}\right\rangle e^{i \lambda_{n} x}, C_{m}^{\mu}(x)\right\rangle C_{m}^{\mu}(x)\right\|_{\infty} \\
& \leq M \max _{1 \leq m \leq M}\left(c \Gamma(\mu)(m+\mu)\left(\frac{2}{\lambda_{ \pm N}}\right)^{\mu-1}\left\|C_{m}^{\mu}(x)\right\|_{\infty}\right) \\
& \leq c M \Gamma(\mu)(M+\mu)\left(\frac{2}{\lambda_{ \pm N}}\right)^{\mu-1} C_{M}^{\mu}(1) .
\end{aligned}
$$

In the last line we use the fact that $C_{m}^{\mu}(x) \leq C_{m}^{\mu}(1)=\frac{\Gamma(m+2 \mu)}{m ! \Gamma(2 \mu)}$ for all $m$, and that $C_{m}^{\mu}(1)$ is an increasing function of $m$ (see $[9$, p.174]). Hence

$$
\left\|P_{M}^{\mu}\left(f-T_{N} f\right)\right\|_{\infty} \leq c \frac{\Gamma(\mu)(M+\mu) \Gamma(M+2 \mu)}{(M-1) ! \Gamma(2 M)}\left(\frac{2}{\lambda_{ \pm N}}\right)^{\mu-1}
$$

as desired.

In fact, since $\left\{\lambda_{n}\right\}$ is assumed to be balanced and $\delta$-separated, we know that $\lambda_{ \pm N}>\delta N$ for all $N$, and we can therefore further 
estimate the truncation error by

$$
\left\|P_{M}^{\mu}\left(f-T_{N} f\right)\right\|_{\infty} \leq c \frac{\Gamma(\mu)(M+\mu) \Gamma(M+2 \mu)}{(M-1) ! \Gamma(2 M)}\left(\frac{2}{\delta N}\right)^{\mu-1} .
$$

Finally, as shown in Lemma 1, if $\left\{\lambda_{n}\right\}$ has uniform density and $N$ is large enough, then we can apply the possibly better estimate of the sum $\sum_{|n|>N}\left|\lambda_{n}\right|^{-\mu}$ to bound the truncation error by

$$
\left\|P_{M}^{\mu}\left(f-T_{N} f\right)\right\|_{\infty} \leq c \frac{\Gamma(\mu)(M+\mu) \Gamma(M+2 \mu)}{(M-1) ! \Gamma(2 M)}\left(\frac{2}{\pi N-t}\right)^{\mu-1} .
$$

Corollary 1 Let $\left\{e^{i \lambda_{n} x}\right\}_{n \in \mathbb{Z}}$ be a frame generated by a balanced sampling sequence. Suppose we are given the first $2 N+1$ frame coefficients of $f \in L^{2}[-1,1]$. If $\mu=\alpha N$ and $M=\beta N$ for appropriate constants $\alpha<1$ and $\beta<1$, then there exists a constant $0<q<1$ such that the truncation error (9) satisfies

$$
\left\|P_{M}^{\mu}\left(f-T_{N} f\right)\right\|_{\infty} \leq c N^{2} q^{N}
$$

Proof This result follows by straightforward arithmetic from Theorem 4. In particular, (28) yields

$$
\left\|P_{M}^{\mu}\left(f-T_{N} f\right)\right\|_{\infty} \leq c \frac{\Gamma(\mu)(M+\mu) \Gamma(M+2 \mu)}{(M-1) ! \Gamma(2 \mu)}\left(\frac{2}{\lambda_{ \pm N}}\right)^{\mu-1} .
$$

Hence, if $\mu=\alpha N$ and $M=\beta N$ for positive constants $\alpha$ and $\beta$, then $\left\|P_{M}^{\mu}\left(f-T_{N} f\right)\right\|_{\infty} \leq c \frac{\Gamma(\alpha N)(\beta+\alpha) N \Gamma((\beta+2 \alpha) N)}{(\beta N-1) ! \Gamma(2 \alpha N)}\left(\frac{2}{\lambda_{ \pm N}}\right)^{\alpha N-1}$.

Furthermore, for $x \geq 1$, the well-known Stirling formula gives

$$
\Gamma(x+1) \leq \sqrt{2 \pi} x^{x+\frac{1}{2}} e^{-x} e^{\frac{1}{12}}
$$

and

$$
\Gamma(x+1) \geq \sqrt{2 \pi} x^{x+\frac{1}{2}} e^{-x} .
$$


Therefore,

$$
\left\|P_{M}^{\mu}\left(f-T_{N} f\right)\right\|_{\infty} \leq c N^{2} q^{N}
$$

where either

$$
\begin{aligned}
q & =\frac{(\beta+2 \alpha)^{\beta+2 \alpha}}{(2 \pi e)^{\alpha} \alpha^{\alpha} \beta^{\beta}}\left(\frac{\pi N}{\lambda_{ \pm N}}\right)^{\alpha} \\
q & =\frac{(\beta+2 \alpha)^{\beta+2 \alpha}}{(2 \pi e)^{\alpha} \alpha^{\alpha} \beta^{\beta}}\left(\frac{\pi}{\delta}\right)^{\alpha} \\
q & =\frac{(\beta+2 \alpha)^{\beta+2 \alpha}}{(2 \pi e)^{\alpha} \alpha^{\alpha} \beta^{\beta}}\left(\frac{N}{N-t}\right)^{\alpha}
\end{aligned}
$$

using estimates (27), (28), and (29) respectively. Notice that the second of these possible values for $q$ is the worst-case scenario. However, if we set $\alpha=\beta=\frac{\delta}{2}$, then in this case we have

$$
q=\left(\frac{3}{2 \sqrt{e}}\right)^{\delta} \approx(0.90)^{\delta}<1
$$

It is important to note that although spectral reprojection is proposed for (nonperiodic) analytic functions, no smoothness conditions are imposed in the above theorems. The assumption of analyticity is necessary in order to control the regularization error (the convergence rate of the true Gegenbauer approximation to the underlying function), as defined in (9). If the function in question is indeed analytic, then the regularization error is exponentially small. For more information on the conditions for exponential convergence of the regularization error of the Gegenbauer reconstruction method, see [14].

\subsection{Extension to piecewise-analytic functions}

While the results in Section 5.2 are given only for nonperiodic analytic functions, they are easily extended to piecewise-analytic functions by treating each region of analyticity separately. To see this, let $f \in L^{2}[-1,1]$ be piecewise-analytic, and assume that $f$ is smooth on the interval $[a, b]$. Define $\epsilon=\frac{b-a}{2}$ and $\delta=\frac{b+a}{2}$. Now notice that for $\xi \in[-1,1], f(x(\xi))=f(\epsilon \xi+\delta)$ is an analytic and nonperiodic 
function in $L^{2}[-1,1]$, and therefore we can implement the Gegenbauer reconstruction as described in Section 5.2, using the partial frame approximation $\left(T_{N} f\right)(\epsilon \xi+\delta)$. Since Theorem 4 proves that the truncation error in each interval of analyticity decays exponentially, we maintain exponential accuracy overall. There is some constant variation in this error estimate. Details for the spectral reprojection method for piecewise-analytic functions given (uniform) Fourier coefficients can be found in [14].

\section{$6 \quad$ Numerical Results}

In what follows we give a variety of numerical results that have been obtained using reprojection techniques to enhance the reconstruction of piecewise-analytic functions using Fourier frame data. We are particularly interested in the case where the sampling sequence is jittered off of the standard sampling sequence, with $\lambda_{n}=n+\nu$ with $\nu$ a uniformly-distributed random variable, as described in (12) and (13). Kadec's 1/4-Theorem proves that if $\inf _{n \in \mathbb{Z}}\left|\lambda_{n}-n\right|<$ $1 / 4$, then the family $\left\{e^{i \pi \lambda_{n} x}\right\}_{n \in \mathbb{Z}}$ forms a Riesz basis for $L^{2}[-1,1]$, which is a stronger condition than a frame [5, p. 163]. Also of interest is the case where the sampling sequence is log-spaced, (11). In fact, such a sampling sequence does not even generate a frame, since the sparse sampling at high frequencies keeps the corresponding family of exponentials from being complete. However, numerical evidence indicates that the spectral reprojection method still delivers improved reconstructions even for "poor" sampling sequences. The log-spaced sampling sequence is also important since it is the onedimensional analogue of a spiral, which is of great interest in the MR imaging community.

When constructing the finite frame approximation, (19), we used the Chebyshev-accelerated frame algorithm from [17, Thm. 1, p. 3333].

Figures 12 through 19 demonstrate an application of the spectral reprojection method for a range of different functions and sampling sequences. Figures 12, 13, and 14 demonstrate the results of the Gegenbauer reconstruction method for frames, (24), when applied to $f(x)=x$, using log-spaced (11) and jittered sampling schemes, (12) 
and (13), respectively. In all examples, we choose the parameters $\mu$ and $M$ in (24) to minimize the $L_{\infty}$ error. Notice in particular that the results shown in Figures 13 through 14 are similar to those in Figure 2, even though the Fourier data is collected non-uniformly.

Figures 15 through 17 demonstrate the same methods applied to the reconstruction of a piecewise-analytic function

$$
f(x)= \begin{cases}\sin (1.6 \pi x) & \text { if } x<0 \\ -x^{3}+2 x^{2}+1 & \text { if } x \geq 0 .\end{cases}
$$

Figures 18 and 19 show the decay rate of the Gegenbauer coefficients in (23) for fixed $\mu$ and increasing $M$ for $f(x)=x$ and (33) respectively. Exponential decay is achieved for increasing values of $\mu$ and $M$ until numerical roundoff error occurs. This is similar to what is seen in the uniform case, (7), [11], and will be investigated in future work.
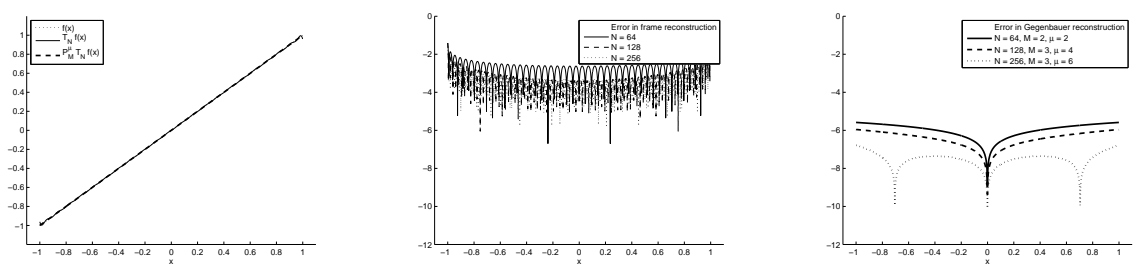

Figure 12: (left) Reconstruction of $f(x)=x$ using $T_{N} f(x)$ in (19) and $P_{M} T_{N} f(x)$ in (24) with a $1 / 4$ jittered sampling scheme (12). (middle) $\log _{10}\left|f(x)-T_{N} f(x)\right|$. (right) $\log _{10}\left|f(x)-P_{M} T_{N} f(x)\right|$. We used $N=64,128$, and 256, with $M=2,3,3$ and $\mu=2,4,6$, respectively. 

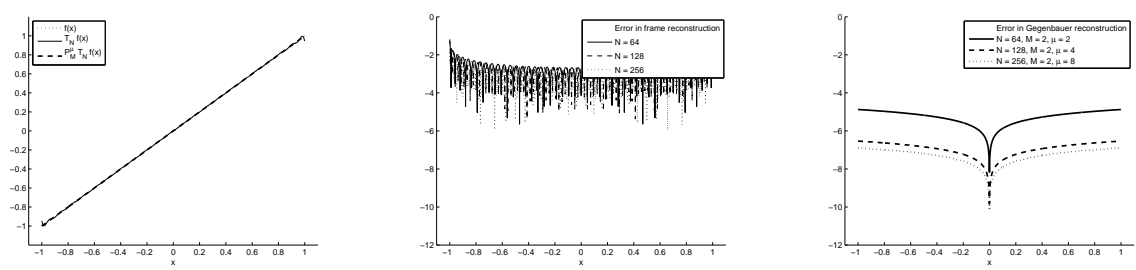

Figure 13: (left) Reconstruction of $f(x)=x$ using $T_{N} f(x)$ in (19) and $P_{M} T_{N} f(x)$ in (24) with a $1 / 2$ jittered sampling scheme (13). (middle) $\log _{10}\left|f(x)-T_{N} f(x)\right|$. (right) $\log _{10}\left|f(x)-P_{M} T_{N} f(x)\right|$. We used $N=64,128$, and 256, with $M=2,2,2$ and $\mu=2,4,8$, respectively.
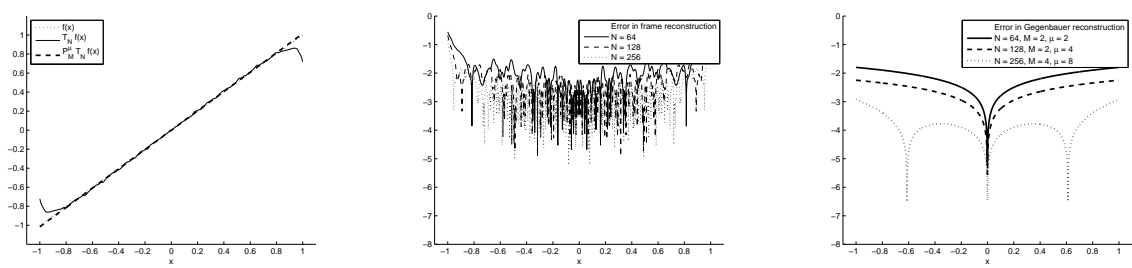

Figure 14: (left) Reconstruction of $f(x)=x$ using $T_{N} f(x)$ in (19) and $P_{M} T_{N} f(x)$ in (24) with a log-spaced sampling scheme (11). (middle) $\log _{10}\left|f(x)-T_{N} f(x)\right|$. (right) $\log _{10}\left|f(x)-P_{M} T_{N} f(x)\right|$. We used $N=64,128$, and 256, with $M=2,2,4$ and $\mu=2,4,8$, respectively. 

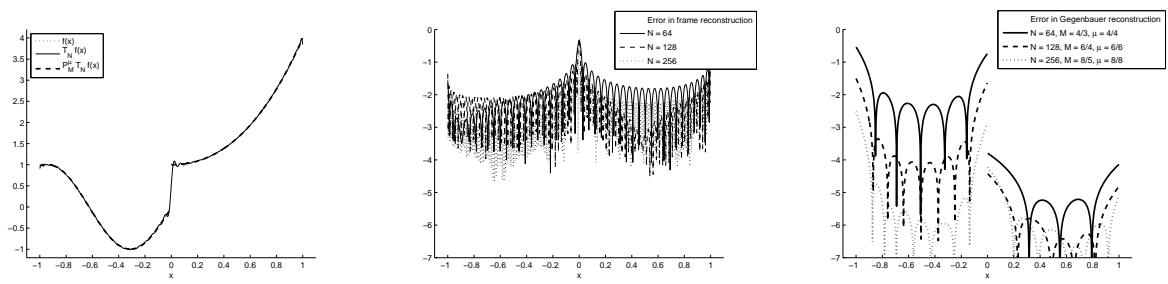

Figure 15: (left) Reconstruction of (33) using $T_{N} f(x)$ in (19) and $P_{M} T_{N} f(x)$ in (24) with a $1 / 4$ jittered sampling scheme (12). (middle) $\log _{10}\left|f(x)-T_{N} f(x)\right|$. (right) $\log _{10}\left|f(x)-P_{M} T_{N} f(x)\right|$. In this simulation we used $N=64,128$ and 256. In the left interval of analyticity, we used $\mu=M=4,6,8$ respectively, while in the right-hand interval of analyticity we used $\mu=4,6,8$ and $M=3,4,5$.
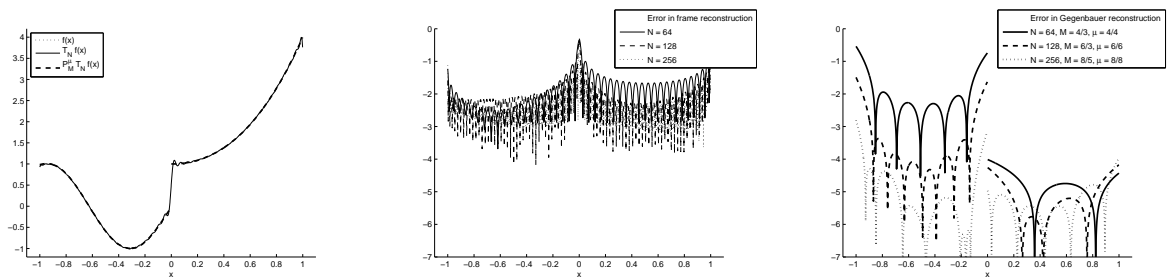

Figure 16: (left) Reconstruction of (33) using $T_{N} f(x)$ in (19) and $P_{M} T_{N} f(x)$ in (24) with a $1 / 2$ jittered sampling scheme (13). (middle) $\log _{10}\left|f(x)-T_{N} f(x)\right|$. (right) $\log _{10}\left|f(x)-P_{M} T_{N} f(x)\right|$. In this simulation we used $N=64,128$ and 256. In the left interval of analyticity, we used $\mu=M=4,6,8$ respectively, while in the right-hand interval of analyticity we used $\mu=4,6,8$ and $M=3,3,5$. 

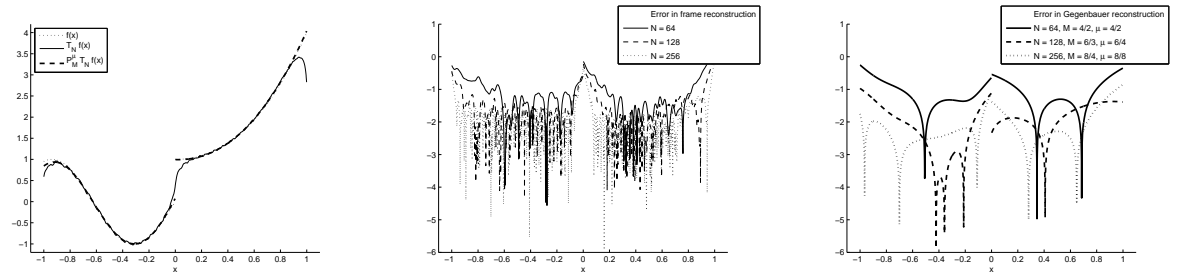

Figure 17: (left) Reconstruction of (33) using $T_{N} f(x)$ in (19) and $P_{M} T_{N} f(x)$ in (24) with a log-spaced sampling scheme (11). (middle) $\log _{10}\left|f(x)-T_{N} f(x)\right|$. (right) $\log _{10}\left|f(x)-P_{M} T_{N} f(x)\right|$. In this simulation we used $N=64,128$ and 256. In the left interval of analyticity, we used $\mu=M=4,6,8$ respectively, while in the right-hand interval of analyticity we used $\mu=2,4,8$ and $M=2,3,4$.
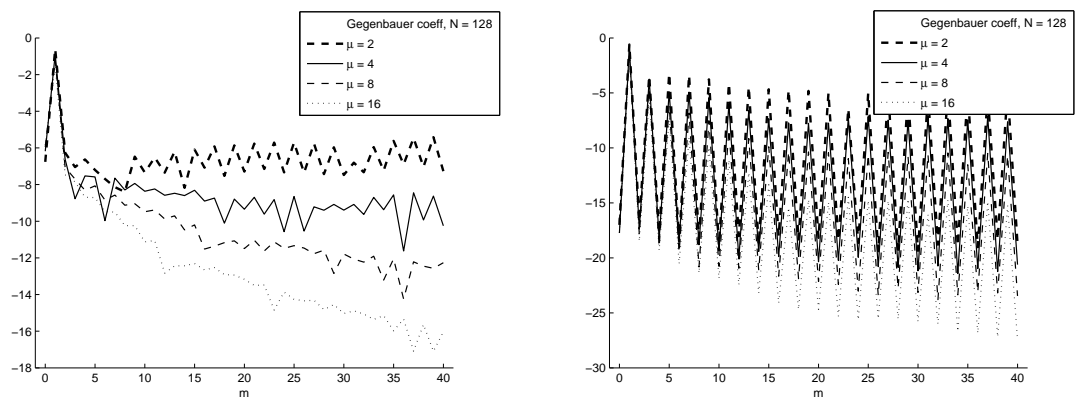

Figure 18: The Gegenbauer coefficients $\log _{10}\left(\left|{\widehat{T_{N} f}}^{\mu}(m)\right|\right),(23)$, for $f(x)=x$ and $N=128$ (left) with jittered sampling, (12), and (right) with log-spaced sampling, (11). 

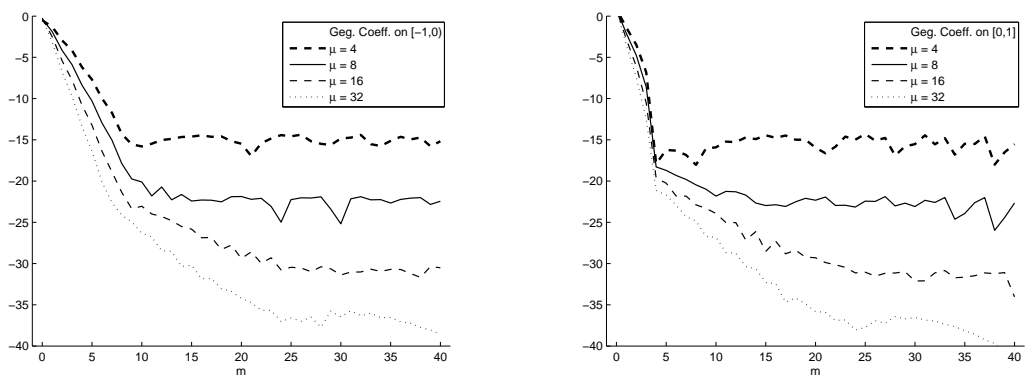

Figure 19: (left) The Gegenbauer coefficients $\log _{10}\left(\left|\widehat{\left.T_{N} f\right)^{\mu}}(m)\right|\right)$, (23), for the first interval of analyticity of $f$ defined by (33). (right) The Gegenbauer coefficients $\log _{10}\left(\left|\widehat{\left.T_{N} f\right)^{\mu}}(m)\right|\right),(23)$, for the second interval of analyticity of $f$ defined by (33). Here $N=128$ with jittered sampling, (12). 


\section{Remarks}

This paper addresses the issue of reconstructing piecewise-analytic functions from a non-uniform sampling sequence of Fourier coefficients. Besides the problems associated with the Gibbs phenomenon $(\mathcal{O}(1))$ oscillations near discontinuities and $\mathcal{O}(1 / N)$ accuracy overall), the sequence $\left\{e^{i \lambda_{n} x}\right\}_{n=-N}^{N}$ does not necessarily form an orthonormal basis.

To resolve these issues, this paper adopts the use of Fourier frames. The general theory of frames makes it possible to employ the spectral reprojection technique $[15,14]$ to mitigate the impact of the Gibbs phenomenon and other undesirable effects of the non-uniform sampling. In particular, Theorem 4 proves that the spectral reprojection error of the Fourier frame approximation onto Gegenbauer polynomials is exponentially small. Numerical evidence presented in Section 6 confirms these results and further indicates that the techniques can be successfully applied to sampling sequences that do not form frames. This is particularly useful in applications such as MRI, where the spiral sampling patterns sometimes used do not constitute frames. Moreover, even when Fourier data is ostensibly collected uniformly, sensitivity in the sensing equipment will undoubtedly lead to a jittered sampling sequence. Due to this sensitivity, it would be worthwhile to investigate the robustness of spectral reprojection for applications where noise is relevant. In particular, other reprojection bases, such as the Freud polynomial bases introduced in [12] may be better suited.

Finally, it would be of interest to extend these results to other frames, e.g. frames of translates and Gabor frames, [5], which will also yield similar behavior for piecewise-analytic functions. In particular, we expect the theory of frame localization, $[1,18]$, to be central.

Acknowledgement 1 This work was supported in part by NSFDMS-FRG award 0652833. 


\section{References}

[1] Radu Balan, Peter G. Casazza, Christopher Heil, and Zeph Landau. Density, overcompleteness, and localization of frames. I. Theory. J. Fourier Anal. Appl., 12(2):105-143, 2006.

[2] J. J. Benedetto and H. C. Wu. Non-uniform sampling and spiral mri reconstruction. Proc. S.P.I.E., 4119(1):130-141, 2000.

[3] John P. Boyd. Chebyshev and Fourier spectral methods. Dover Publications Inc., Mineola, NY, second edition, 2001.

[4] Ole Christensen. Finite-dimensional approximation of the inverse frame operator. J. Fourier Anal. Appl., 6(1):79-91, 2000.

[5] Ole Christensen. An introduction to frames and Riesz bases. Applied and Numerical Harmonic Analysis. Birkhäuser Boston Inc., Boston, MA, 2003.

[6] Ole Christensen and Alexander M. Lindner. Frames of exponentials: lower frame bounds for finite subfamilies and approximation of the inverse frame operator. Linear Algebra Appl., 323(1-3):117-130, 2001.

[7] R. J. Duffin and A. C. Schaeffer. A class of nonharmonic Fourier series. Trans. Amer. Math. Soc., 72:341-366, 1952.

[8] Knut S. Eckhoff. Accurate reconstructions of functions of finite regularity from truncated Fourier series expansions. Math. Comp., 64(210):671-690, 1995.

[9] Arthur Erdélyi, Wilhelm Magnus, Fritz Oberhettinger, and Francesco G. Tricomi. Higher transcendental functions. Vol. II. Robert E. Krieger Publishing Co. Inc., Melbourne, Fla., 1981. Based on notes left by Harry Bateman, Reprint of the 1953 original.

[10] James Geer and Nana Saheb Banerjee. Exponentially accurate approximations to piece-wise smooth periodic functions. J. Sci. Comput., 12(3):253-287, 1997. 
[11] Anne Gelb. Parameter optimization and reduction of round off error for the Gegenbauer reconstruction method. J. Sci. Comput., 20(3):433-459, 2004.

[12] Anne Gelb and Jared Tanner. Robust reprojection methods for the resolution of the Gibbs phenomenon. Appl. Comput. Harmon. Anal., 20(1):3-25, 2006.

[13] David Gottlieb and Steven A. Orszag. Numerical analysis of spectral methods: theory and applications. Society for Industrial and Applied Mathematics, Philadelphia, Pa., 1977. CBMS-NSF Regional Conference Series in Applied Mathematics, No. 26.

[14] David Gottlieb and Chi-Wang Shu. On the Gibbs phenomenon and its resolution. SIAM Rev., 39(4):644-668, 1997.

[15] David Gottlieb, Chi-Wang Shu, Alex Solomonoff, and Hervé Vandeven. On the Gibbs phenomenon. I. Recovering exponential accuracy from the Fourier partial sum of a nonperiodic analytic function. J. Comput. Appl. Math., 43(1-2):81-98, 1992. Orthogonal polynomials and numerical methods.

[16] David Gottlieb and Eitan Tadmor. Recovering pointwise values of discontinuous data within spectral accuracy. In Progress and supercomputing in computational fluid dynamics (Jerusalem, 1984), volume 6 of Progr. Sci. Comput., pages 357375. Birkhäuser Boston, Boston, MA, 1985.

[17] Karlheinz Gröchenig. Acceleration of the frame algorithm. IEEE Trans. SSP, 41/12:3331-3340, 1993.

[18] Karlheinz Gröchenig. Localization of frames, Banach frames, and the invertibility of the frame operator. J. Fourier Anal. Appl., 10(2):105-132, 2004.

[19] Jan S. Hesthaven, Sigal Gottlieb, and David Gottlieb. Spectral methods for time-dependent problems, volume 21 of Cambridge Monographs on Applied and Computational Mathematics. Cambridge University Press, Cambridge, 2007.

[20] S. Jaffard. A density criterion for frames of complex exponentials. Michigan Math. J., 38(3):339-348, 1991. 
[21] J G Pipe and P Menon. Sampling density compensation in mri: rationale and an iterative numerical solution. Magn Reson Med, 41(1):179-86, 1999.

[22] Bernie D. Shizgal and Jae-Hun Jung. Towards the resolution of the Gibbs phenomena. J. Comput. Appl. Math., 161(1):41-65, 2003.

[23] Alex Solomonoff. Reconstruction of a discontinuous function from a few Fourier coefficients using Bayesian estimation. $J$. Sci. Comput., 10(1):29-80, 1995.

[24] Eitan Tadmor and Jared Tanner. Adaptive mollifiers for high resolution recovery of piecewise smooth data from its spectral information. Found. Comput. Math., 2(2):155-189, 2002.

[25] Adityavikram Viswanathan. Spectral sampling and discontinuity detection methods with application to magnetic resonance imaging. Master's thesis, Arizona State University, Tempe, Arizona, May 2008.

[26] Adityavikram Viswanathan, Anne Gelb, Douglas Cochran, and Rosemary Renaut. On reconstruction from non-uniform spectral data. J. Sci. Comput., 45(1-3):487-513, 2010. 